Check for updates

Cite this: Mater. Adv., 2021, 2, 4733

Received 30th March 2021, Accepted 29th May 2021

DOI: 10.1039/d1ma00285f

rsc.li/materials-advances

\section{An in situ gelling BMSC-laden collagen/silk fibroin double network hydrogel for cartilage regeneration $\dagger$}

\begin{abstract}
Yajie Zhang, (D) ${ }^{a}$ Min Liu (D) ${ }^{b}$ and Renjun Pei ${ }^{\star a}$
Collagen (Col)-based injectable hydrogels are desirable scaffolds for cartilage tissue engineering. However, fabrication of Col-based injectable hydrogels with short gelation time, great biocompatibility and good mechanical properties still faces great challenges. In this work, an injectable bone marrow mesenchymal stem cell (BMSC)-encapsulated double network (DN) hydrogel was fabricated via collagen (Col), silk fibroin (SF) and poly(ethylene glycol) (PEG), in which PEG-Tetrazine (Tz) and Col-Norbornene $\mathrm{Nb}$ ) were used to crosslink through bioorthogonal reactions to form the first network rapidly, and ultrasonic induction was carried out to form the second SF network. The experimental results showed that the mechanical properties of the Col-PEG/SF DN hydrogel were obviously enhanced compared to those of Col-PEG or SF single network hydrogels. In vivo assays demonstrated that the Col-PEG/SF DN hydrogel could significantly repair full-thickness cartilage defects generated in a rat model after 8 weeks of implantation. These results suggested that the Col-PEG/SF DN hydrogel possesses excellent properties and is a potential treatment candidate for cartilage tissue engineering.
\end{abstract}

\section{Introduction}

Articular cartilage with its unique biomechanical properties is vital for the almost frictionless movement of articulating joints. ${ }^{1}$ Unfortunately, cartilage repairs are greatly limited on its own due to its avascular nature. ${ }^{2-4}$ Currently, several medical treatments are available to cure large chondral lesions, including microfracture (MF), osteochondral transplant (OCT), and autologous chondrocytes implantation (ACI). However, these treatments still have several drawbacks, such as donor site morbidity, graft hypertrophy, and inconsistent repair tissue. ${ }^{5,6}$ Recently, cartilage tissue engineering (CTE) has been attractive for repairing damaged cartilage by implanting a native cartilagemimicking construct which consists of cells, bioscaffolds, and growth factors. ${ }^{7-10}$ Meanwhile, bone marrow mesenchymal stem cells (BMSCs) have attracted the most attention in CTE, because they could be isolated and expanded relatively easily from various tissues and possess excellent homing ability, proliferation and multi-lineage differentiation potential. ${ }^{11-13}$ Furthermore, transforming growth factor- $\beta$ (TGF- $\beta$ ), a short-lived cytokine, could

\footnotetext{
${ }^{a}$ CAS Key Laboratory for Nano-Bio Interface, Suzhou Institute of Nano-Tech and Nano-Bionics, Chinese Academy of Sciences, Suzhou, 215123, China. E-mail: rjpei2011@sinano.ac.cn

${ }^{b}$ Institute for Interdisciplinary Research, Jianghan University, Wuhan 430056, China

$\dagger$ Electronic supplementary information (ESI) available. See DOI: 10.1039/ d1ma00285f
}

trigger the chondrogenesis of MSCs and activate the synthesis of proteoglycans and Type II collagen, which plays an important role in both natural cartilage and approaches to engineer artificial cartilage. $^{14}$

Type I collagen (Col) is the main component of a cartilage extra cellular matrix (ECM), and is widely used in commercial CTE scaffolds undergoing clinical trial (e.g. NOVOCART ${ }^{\circledR} 3 \mathrm{D}$, CaReS $^{\circledR}$, NeoCart ${ }^{\circledR}$ and MACI), owing to its intrinsic biocompatibility, bioactivity and vast safety approvals. ${ }^{15}$ Many studies have reported that $\mathrm{Col}$ has the ability to promote MSC proliferation, and enhance their homing and chondrogenic differentiation. ${ }^{16}$ However, Col still has several disadvantages, such as poor solubility and difficulty in modifying it chemically, which greatly limit the preparation of Col-scaffolds in CTE. Traditionally, cells are either seeded on the pre-formed Col-scaffolds, in which the spatial distribution and location of cells could not be controlled, or pre-mixed with the acidextracted and then neutralized Col solutions, and cell-laden Col-scaffolds are then fabricated by self-assembly of Col solutions at physiological $\mathrm{pH}$ and temperature, which was neither timesaving nor biofriendly. Thereafter, the prepared cell-scaffolds usually were cut to match the defect shapes and then implanted, which was a waste of time and also led to size mismatch. ${ }^{17-20}$ Currently, among all the bioscaffolds, injectable hydrogels have received widespread attention due to their high water content, 3D porous framework, minimally invasive injection method and could form any desired shape according to the defects. ${ }^{21,22}$ 
Therefore, constructing ideal injectable Col hydrogels will provide a promising therapeutic platform in CTE. Unfortunately, it is still a great challenge to fabricate injectable Col hydrogels that can undergo in situ gelation with both suitable gelation rate and great biocompatibility. In this study, water-soluble Col-succinic anhydride (SAH) was synthesized for further modification to obtain the precursor of injectable hydrogels. Nevertheless, injectable hydrogels with conventional preparation, for example ionic crosslinking, pH/thermal crosslinking, photopolymerization, the $\mathrm{Cu}(\mathrm{I})$ catalyzed 1,3-dipolar cycloaddition reaction (CuAAC), Michael-type addition, enzyme-mediated crosslinking and Schiff base formation, have unstable mechanical properties, a slow gelation rate or certain cytotoxicity. ${ }^{23,24}$ To overcome the above-mentioned challenges, bioorthogonal reactions with great biocompatibility and fast reaction rates under physiological conditions have already been carried out as an alternative method to fabricate fast-forming injectable hydrogels with controllable mechanical properties. $^{25,26}$ Particularly, the bioorthogonal inverse electron demand Diels-Alder click reaction between tetrazine $(\mathrm{Tz})$ and norbornene $(\mathrm{Nb})$ has high reaction kinetics and chemoselectivity, but does not need any additional additives, initiators or catalysts. In addition, it is easy for $\mathrm{Nb}$ to undergo chemical reactions with Col-SAH in aqueous media because of its good water-solubility, and is superior to many other waterinsoluble bioorthogonal moieties. ${ }^{27,28}$ Thus, the bioorthogonal reaction between $\mathrm{Tz}$ and $\mathrm{Nb}$ emerged as an attractive way to prepare hydrogels owing to their great biocompatibility, short gelation time and relatively simple synthetic procedures to obtain the precursors.

Another challenge for Col hydrogels in CTE is the inferior mechanical properties. ${ }^{7,29}$ Traditional hydrogels consisting of a single polymer network generally possess weak mechanical properties which are much inferior to those of normal cartilage tissues. In contrast, hydrogels based on double networks (DNs) comprise two separate crosslinked networks with significantly opposite mechanical properties and exhibit an efficient energy dissipation mechanism. ${ }^{30,31}$ Consequentially, the mechanical properties of DN hydrogels tend to be superior to those of individual component networks, which reveal crucial promise in the design of hydrogels for cartilage regeneration. Hence, a DN hydrogel system with stronger mechanical properties was developed for cartilage injury repair.

Silk fibroin (SF) is an important natural protein with great biocompatibility, which could support the proliferation of MSCs and guide them toward chondrogenic differentiation. ${ }^{16,32}$ Importantly, silk fibroin has excellent mechanical properties due to the formation of the $\beta$-sheet structure. ${ }^{3,34}$ Our previous studies have already reported that BMSC-encapsulated SF hydrogels could be obtained without any chemical treatment but by simply ultrasonically induced gelation. ${ }^{35}$ As mentioned above, the SF hydrogel could easily integrate with the above mentioned Col hydrogel to develop an in situ gelling BMSC-encapsulated DN hydrogel with a suitable gelation rate, great biocompatibility and good mechanical properties for cartilage regeneration.

Herein, an injectable BMSC-encapsulated DN hydrogel based on Col and SF was explored. Briefly, Col and PEG were modified with $\mathrm{Nb}$ and $\mathrm{Tz}$ which could crosslink within several seconds and set as the first network. Ultrasonic induced SF then could crosslink through the $\beta$-sheet structure and sets as the second network. Thereafter, the gelation rate and physiochemical properties of this system were optimized. Simultaneously, BMSCs could be evenly premixed in the precursor solutions and encapsulated in the hydrogel after gelation. Afterwards, the BMSC survival and proliferation were investigated. Finally, the capability of this system for CTE was evaluated in vitro and in vivo, (Scheme 1).

\section{Materials and methods}

\subsection{Materials}

4-Arm poly(ethylene glycol) carboxylic acid (4-arm PEG-CM, $20 \mathrm{KDa}$ ) was supplied by Xiamen Sinopeg Biotech Co., Ltd. Benzotriazol-1-yloxy-tris-pyrrolidinophosphonium hexafluorophosphate (pyBOP), $\mathrm{N}$-hydroxysuccinimide (NHS) and 5-norbornene-2-methylamine $\mathrm{Nb}$ ) were purchased from Tokyo Chemical Industry Co., Ltd. Tetrazine-amine was purchased from Biocone Biotechnology Co., Ltd. 1-Ethyl-3-(3-dimethyllaminopropyl)carbodiimide hydrochloride (EDC) and 2-( $N$-morpholino)ethanesulfonic acid monohydrate (MES) were purchased from Sigma-Aldrich. Transforming growth factor- $\beta 1$ (TGF- $\beta 1$ ) and fibroblast growth factor (FGF) were obtained from PeproTech. Insulin-Transferrin-Selenium (ITS), sodium pyruvate, fetal bovine serum (FBS), Dulbecco's modified Eagle's medium (low glucose) and TrypLE ${ }^{\mathrm{TM}}$ express enzymes were obtained from Gibco (Life Technologies, USA). Col, SF and BMSCs were prepared according to our previous methods. ${ }^{31,33}$ All the other regents were obtained from Sinopharm Chemical Reagent Co. Ltd. and used as received.

${ }^{1} \mathrm{H}$ NMR spectra were collected using a Varian NMR spectrometer at $400 \mathrm{MHz}$. WST assay was conducted using BiotekCytation 3. FTIR spectroscopies were obtained from a Thermo Fisher FTIR spectrometer, and the samples were pelletized with $\mathrm{KBr}$ before measurement. The morphology of the hydrogel was observed under a scanning electron microscope (SEM, Quanta FEG 250). The cellular images were observed using a confocal laser scanning microscope (CLSM, Nikon A1). The storage modulus $\left(G^{\prime}\right)$ and loss modulus $\left(G^{\prime \prime}\right)$ of hydrogels were measured using a Haake rotational rheometer (RS6000).

\subsection{Synthesis of Col-Nb and PEG-Tz}

For the synthesis of Col-Nb, Col-SAH was first prepared according to our previous work. ${ }^{36}$ Briefly, Col was dissolved in $1 \%$ acetic acid under ice-bath conditions. $24 \mathrm{~h}$ later, the $\mathrm{pH}$ of the solution was adjusted to $\sim 10$ followed by adding $\mathrm{SAH}$ dropwise (dissolved in acetone and 15 times equivalent to the weight of $\mathrm{Col}$ ) to the above solution. After being stirred for $24 \mathrm{~h}$ at $4{ }^{\circ} \mathrm{C}(\mathrm{pH} \approx 9)$, the solution was dialyzed (14000 Da) against deionized water at $4{ }^{\circ} \mathrm{C}$ for 3 days, and the obtained water-soluble Col-SAH was lyophilized and stored at $-20{ }^{\circ} \mathrm{C}$. Next, Col-SAH was dissolved in $100 \mathrm{mM}$ MES buffer (pH 5.5) followed by adding EDC and NHS (4 times equivalent to carboxyl groups of $\mathrm{Col}$ ) to activate the carboxyl groups on the backbone of Col. After stirring for $15 \mathrm{~min}, \mathrm{Nb}$ 


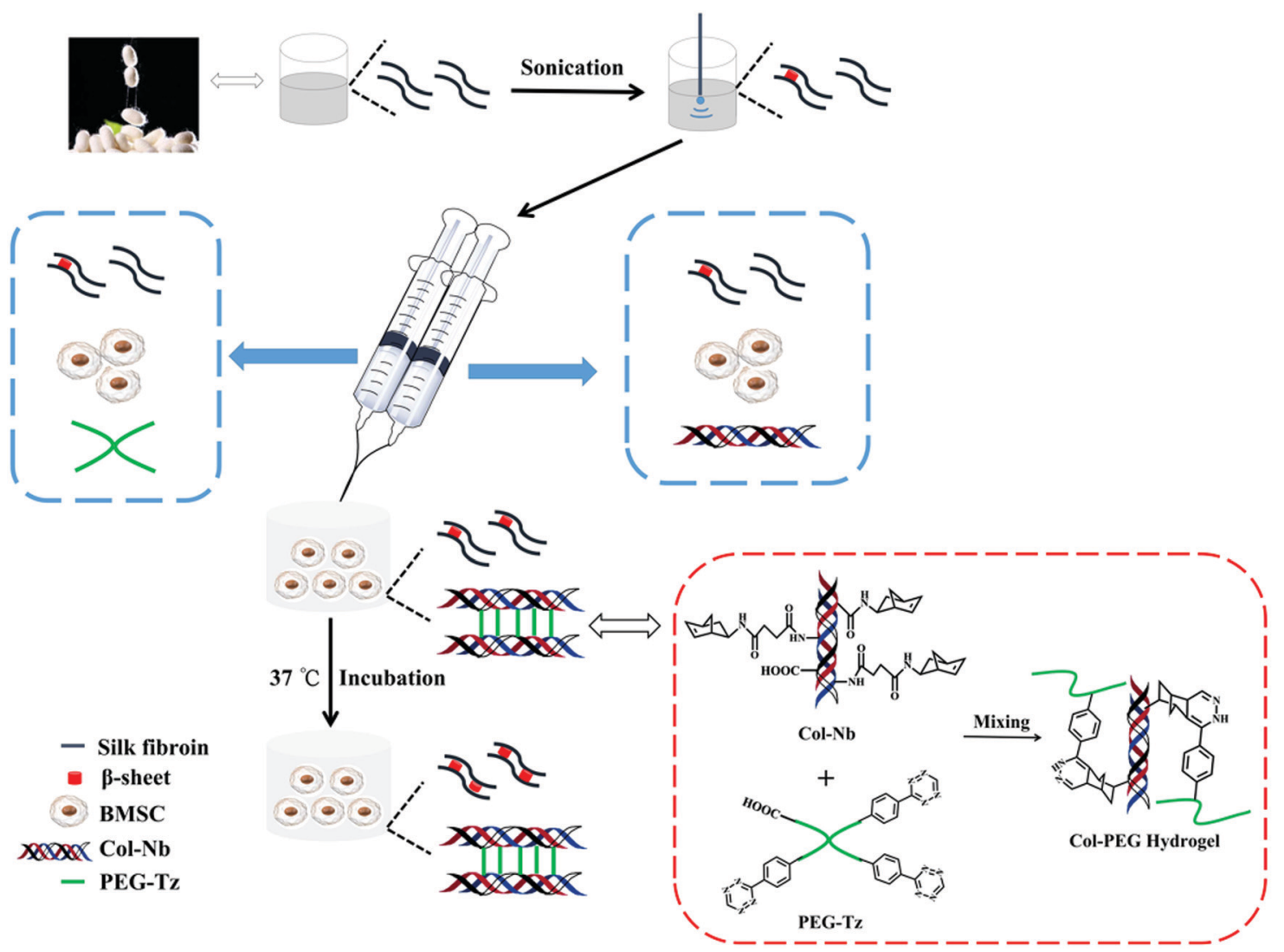

Scheme 1 Preparation of the BMSC-laden injectable Col-PEG/SF hydrogel for cartilage regeneration.

(1.5 times equivalent to the carboxyl groups of $\mathrm{Col}$ ) was added and stirred overnight. Finally, the solution was purified by dialyzing $(\mathrm{MWCO}=3500)$ against deionized water for 3 days and lyophilized to obtain Col-Nb.

For the synthesis of PEG-Tz, 4-arm PEG-COOH was dissolved in dichloromethane followed by adding $N, N$-diisopropylethylamine and pyBOP (4 and 2.5 times equivalent to the carboxyl groups of 4-arm $\mathrm{PEG}-\mathrm{COOH}$, respectively), and the mixture was stirred in an ice bath for $30 \mathrm{~min}$. Tetrazine-amine HCl (1.2 times equivalent to the carboxyl groups of 4-arm PEG-COOH) was then added and the solution was stirred for $24 \mathrm{~h}$. Thereafter, the mixture was precipitated in anhydrous diethyl ether and the crude product was then purified by dialyzing $($ MWCO $=3500)$ against deionized water for 3 days and lyophilized to obtain the final product (PEG-Tz).

\subsection{Preparation of Col-PEG/SF hydrogels}

The compositions of different Col-PEG/SF hydrogels are listed in Table S2 (ESI $\dagger$ ). The weight ratio of Col-Nb to PEG-Tz was determined by the change of the hydrogel color. For the hydrogel preparation, $1 \mathrm{~mL}$ of pure SF $(10 \% \mathrm{w} / \mathrm{v})$ solution was treated under an ultrasonic cell crusher (at 50\% amplitude for $1 \mathrm{~min}, 5 \mathrm{~s}$ ultrasonication followed by a $5 \mathrm{~s}$ pause) to induce $\beta$-sheet formation. Thereafter, the Col-Nb and PEG-Tz were quickly solubilized in SF solution with certain concentrations followed by incubating at $37{ }^{\circ} \mathrm{C}$ for $30 \mathrm{~min}$ before use.
To prepare the BMSC-encapsulated Col-PEG/SF hydrogels, first, Col-Nb and PEG-Tz were dissolved in ultrasonicated SF solution, respectively. Then, BMSCs (passage 2-6, $1.5 \times 10^{6}$ cells per $\mathrm{mL}$ ) were separately mixed with two precursor solutions (finally total concentration: $5 \% \mathrm{w} / \mathrm{v}$ for Col-Nb, $5 \% \mathrm{w} / \mathrm{v}$ for PEG-Tz and $5 \% \mathrm{w} / \mathrm{v}$ for SF). Next, these two precursor solutions were mixed thoroughly. The gelation time was calculated by considering the gel state when the sample was not flowing after inverting the vial. After the formation of the first network of the hydrogel, the hydrogels were maintained at $37{ }^{\circ} \mathrm{C}$ to gel completely.

\subsection{Characterization of hydrogels}

First, FT-IR spectroscopy was carried out to demonstrate the formation of $\beta$-sheets among SF in Col-PEG/SF hydrogels. Second, the structure of the lyophilized hydrogel was observed by SEM after being sputter-coated with gold. Next, rheological tests of the hydrogels were conducted using a Haake rotational rheometer and the compression test was carried out using an Instron 3365. Then, swelling studies were carried out as follows: the freeze-dried hydrogels were weighed as $W_{\mathrm{d}}$. The wet hydrogels also were weighed $\left(W_{\mathrm{w}}\right)$ after immersion in PBS to reach equilibrium. The equation $\left(\left(W_{\mathrm{w}}-W_{\mathrm{d}}\right) / W_{\mathrm{d}}\right)$ was used to calculate the swelling ratio (SR) of hydrogels. Finally, the degradation experiment was performed to analyze the possible degradation rate in vivo. Briefly, hydrogels were lyophilized and weighed $\left(W_{1}\right)$. Next, the hydrogels were incubated in PBS containing degrading enzymes with different concentrations $(0,20$, 
and 200 units per $\mathrm{mL}$ ) at $37{ }^{\circ} \mathrm{C}$, and the lyophilized residual hydrogels were weighed $\left(W_{\mathrm{r}}\right)$ at specific time points. The mass loss of hydrogels was calculated according to the following equation: $\left(\left(W_{1}-W_{\mathrm{r}}\right) / W_{1}\right)$.

\subsection{Proliferation of BMSCs within Col-PEG/SF hydrogels}

The as-prepared hydrogels were immersed in the complete medium (DMEM/F-12 free media with 10\% FBS, supplemented with 100 units per $\mathrm{mL}$ of streptomycin and penicillin) and the media was replaced every 48-72 hours. Finally, water soluble tetrazolium (WST, Beyotime Biotechnology) was used to test the proliferation of BMSCs at different time points. Meanwhile, the cell viability of encapsulated-BMSCs was also assessed by using a Live/Dead staining assay kit (Life Technologies, USA).

\subsection{Chondrogenesis induction of BMSCs within Col-PEG/SF hydrogels in vitro}

Briefly, the BMSC-encapsulated hydrogels were incubated in complete medium for 1 day, and then transferred to the inductive medium containing $50 \mu \mathrm{g} \mathrm{mL} \mathrm{L}^{-1}$-ascorbic acid, $100 \mathrm{nM}$ dexamethasone, $40 \mu \mathrm{g} \mathrm{mL} \mathrm{m}^{-1}$ proline, $1 \times$ ITS, $1 \times$ sodium pyruvate, FGF $\left(10 \mathrm{ng} \mathrm{mL}^{-1}\right)$ and TGF- $\beta 1\left(10 \mathrm{ng} \mathrm{mL}^{-1}\right)$. The hydrogels were taken out at certain times, and rinsed with PBS three times for the following measurement.

\subsection{Chondrogenic specific gene expressions}

The mRNA expression levels of chondrogenic-related genes were then assessed at 0 day (cultured in complete medium for 1 day), 14th day and 28th day by qRT-PCR, including Collagen type II (Col II), Aggrecan (AGG), SRY-type high mobility group box 9 (Sox 9). The relative expressions for the target genes were represented as a fold change to undifferentiated BMSCs (before encapsulation) and normalized to that of the reference gene GAPDH and the primers for qRT-PCR are listed in Table S3 (ESI $\dagger$ ). At each time point, the hydrogels were taken out and rinsed with PBS three times. The total RNA was then extracted using TRIzol $^{\mathrm{TM}}$ Reagent (Invitrogen ${ }^{\mathrm{TM}}$ ) and the

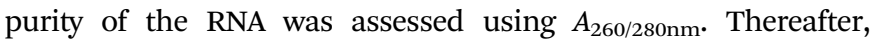
$500 \mathrm{ng}$ of RNA was reverse transcribed into cDNA using the PrimeScript $^{\mathrm{TM}}$ RT Reagent Kit (Takara). qRT-PCR was performed using a Mastercycler ${ }^{\circledR}$ nexus (Eppendorf) using a SYBR Green I PCR Kit.

\subsection{Immunohistochemical analysis in vitro}

To further confirm the chondrogenic differentiation capacity of BMSCs within Col-PEG/SF hydrogels in vitro, the hydrogels were taken out at each time point to perform immunohistochemical analysis. In brief, samples were fixed in $4 \% \mathrm{w} / \mathrm{v}$ paraformaldehyde overnight followed by dehydration and slicing. Immunohistology was conducted using the following primary antibodies: anti-AGG (1:200, Servicebio) and anti-Col II $\left(1: 100\right.$, Servicebio) at $4{ }^{\circ} \mathrm{C}$ overnight. The samples were then incubated with the secondary antibody ( $1: 300$, Servicebio) for $1 \mathrm{~h}$, followed by washing with PBS 3 times. After rinsing in PBS, the samples were incubated with DAPI stain for $10 \mathrm{~min}$. Finally, the slides were examined using a confocal laser scanning microscope. The obtained BMSC-laden Col-PEG/SF hydrogels cultured in complete medium for 1 day were set as controls.

\subsection{Fabrication of articular cartilage defects}

All animal experiments were conducted in accordance with the relevant laws and institutional guidelines following the approval of the Ethics Committee of Suzhou Institute of Nano-Tech and Nano-Bionics, Chinese Academy of Sciences. Eighteen healthy female Sprague-Dawley rats weighting 260$280 \mathrm{~g}$ obtained from Nanjing Sikerui Biological Technology Co., Ltd. were selected randomly for the experiment, and acclimated under pathogen-free conditions for 1 week. Thereafter, the rats were divided into 3 groups: control group, hydrogel group and BMSC-TGF- $\beta 1$-laden hydrogel $(\mathrm{B}+\mathrm{T}$ hydrogel) group. All the rats were anesthetized with $50 \mathrm{mg} \mathrm{kg}^{-1}$ pentobarbital sodium via intraperitoneal injection, and then the hair on the joints of rats was removed. Subsequently, the anesthetized rats were placed in a supine position. The operation was carried out in the center of the condyle, and a full-thickness defect with the size of $3 \mathrm{~mm} \times 3 \mathrm{~mm}$ (diameter $\times$ depth) was created using a drill. After irrigating with physiological saline, the holes were carefully dried. Then, the incision was then sutured for the control group, while for the experimental group, the hydrogel with/without BMSCs + TGF- $\beta 1$ was quickly injected into the hole before solidification, and the incision was then sutured. After the operation, the rats were administered with antibiotics for 3 days, and had free access to food and water. Finally, the rats were sacrificed and the condyles were collected after feeding for 8 weeks.

\subsection{Macroscopic, histological and immunohistochemical analysis in vivo}

After sacrifice, the obtained condyles were assessed for cartilage repair according to the modified Wakitani's histological assessment scores (Table S4, ESI $\dagger$ ), and then fixed in $4 \%$ paraformaldehyde for $24 \mathrm{~h}$. After rinsing with PBS, the condyles were subsequently decalcified in PBS containing 15\% EGTA at $4{ }^{\circ} \mathrm{C}$, and the solution was changed every 4 days. 2 months later, the tissue was washed with water followed by progressive replacement with a graded ethanol series. Thereafter, the samples were processed for embedding in paraffin and cut into 4-6 $\mu \mathrm{m}$ sections. To evaluate the morphology of the regenerated cartilage, hematoxylin and eosin (H\&E), safranin-O staining and toluidine blue staining were used according to the standard procedure.

\subsection{Statistical analysis}

All the experimental results were reported as mean \pm standard deviation for in vitro and in vivo studies. All experiments were performed with at least three replicates. The statistical data analysis was conducted using the Origin Pro 8.5 program and $p$ values $<0.05$ were considered statistically significant. ${ }^{*} p<$ $0.05,{ }^{* *} p<0.01,{ }^{* *} p<0.001$. 


\section{Results and discussion}

\subsection{Characterization of hydrogels}

Although numerous tough hydrogels have been reported, they almost have some shortcomings such as the poor biocompatibility caused by the application of toxic crosslinking agents and timeconsuming gelation methods. In this research, we explored a bio-friendly method to synthesize Col-PEG/SF hydrogels with a fast gelation rate and superior mechanical properties, which could be utilized as the engineered cartilage tissue constructs in vivo. Specifically, bioorthogonal pairs were modified onto Col and PEG which allowed the Col-PEG crosslinking within several seconds. Natural Col is a promising biomaterial in tissue engineering but limited by its poor water solubility. To address this limitation, the amine groups of Col were transferred into carboxyl groups using SAH, and the obtained Col-SAH showed excellent water solubility. Thereafter, Col-Nb was then synthesized through an EDC/NHS activated reaction, which was extremely optimized for the fabrication method of the Col-based injectable hydrogel. The ${ }^{1} \mathrm{H}$ NMR spectra (Fig. S1, ESI $\dagger$ ) confirmed the successful synthesis of Col-Nb and PEG-Tz conjugates. Due to the complex structure of Col, the DS of Col$\mathrm{Nb}$ could hardly be calculated, while the DS of PEG-Tz was determined to be $\sim 95 \%$.

To optimize the Col/PEG ratios of Col-PEG hydrogels, different hydrogels were prepared (ESI, $\dagger$ Table $\mathrm{S} 1, \mathrm{Col}_{1}-\mathrm{PEG}_{2}$, $\mathrm{Col}_{1}-\mathrm{PEG}_{1}$ and $\mathrm{Col}_{2}-\mathrm{PEG}_{1}$ ). As shown in Fig. S2 (ESI $\left.\dagger\right)$, there were few bubbles within the gels, which were the unique byproduct (nitrogen) produced during the crosslinking reaction.
The $\mathrm{Col}_{1}-\mathrm{PEG}_{1}$ hydrogel was completely gelled because the pink colour of the hydrogel almost disappeared resulting from residual Tz. Therefore, the ratio of Col to PEG was finally determined to be $1: 1$ in the following work. Under this condition, the hydrogel could form immediately, around $10 \mathrm{~s}$, which was extremely faster than that for almost all reported Col-scaffolds and showed good injectability. Meanwhile, the SF hydrogel is formed via the formation of $\beta$-sheets among ultrasonically induced SF. Therefore, FT-IR was commonly performed to analyse whether the $\beta$-sheet was successfully formed among the ultrasonically induced SF. As shown in Fig. S3 (ESI $\dagger$ ), the FT-IR spectra of the Col-PEG/SF hydrogel showed a strong peak at $1622 \mathrm{~cm}^{-1}$ (amide I) and another characteristic peak was at $1515 \mathrm{~cm}^{-1}$ (amide II), which were assigned to the $\beta$-sheet crystalline structure. ${ }^{37-39}$

The greatest challenge for hydrogels in CTE is the mismatching mechanical strength of native tissue, which neither possesses enough mechanical strength for withstanding physiologic loads during joint movement nor provides sufficient biomechanical stimulation of 3D-encapsulated cells, and thereby result in the failure of cartilage repair and reconstruction. ${ }^{11,22,40}$ As expected, the Col-PEG/SF hydrogels exhibited excellent mechanical properties, which were much superior to those of Col-PEG or SF hydrogels. To optimize the physicochemical properties of the Col-PEG/SF hydrogels, different hydrogels were prepared (ESI, $\uparrow$ Table S2, $\mathrm{PEG}_{7.5}-\mathrm{Col}_{7.5}, \mathrm{Col}_{5}-\mathrm{PEG}_{5} / \mathrm{SF}_{5}$ and $\left.\mathrm{Col}_{5}-\mathrm{PEG}_{5} / \mathrm{SF}_{10}\right)$. As shown in Fig. 1A, as the SF content increased in the hydrogel system and the stiffness of the hydrogel significantly increased. Therefore, $\mathrm{Col}_{5}-$ $\mathrm{PEG}_{5} / \mathrm{SF}_{10}$ was determined to be used in the following experiments
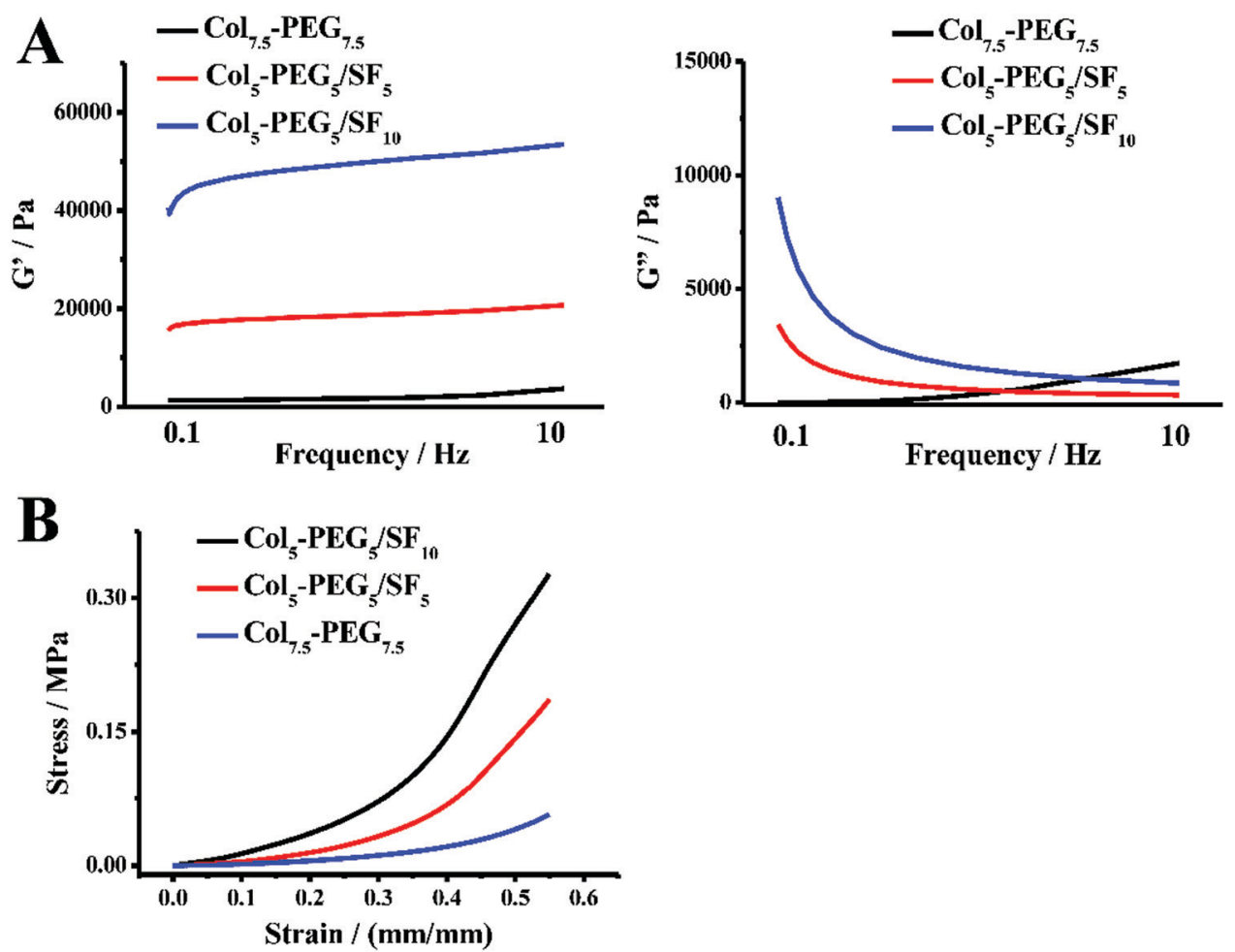

Fig. 1 Rheological test of the hydrogels, $G^{\prime}$ : storage modulus; $G^{\prime \prime}$ : loss modulus (A); compression stress-strain curves of hydrogels (B). 
which possessed the highest storage modulus among the test groups. Similarly, Fig. 1B illustrates that the Col-PEG/SF hydrogels withstood much stronger anti-compressive stress than pure PEG hydrogels. Cyclic compressive stress-strain curves (Fig. 2A) showed that the recoverability of the PEG hydrogels was decreased after combining with the SF hydrogel, and with the increase of SF content, the Col-PEG/SF hydrogels showed worse recoverability. Fig. $2 \mathrm{~B}$ shows that $\mathrm{Col}_{5}-\mathrm{PEG}_{5} / \mathrm{SF}_{5}$ could recover to its original shape after the release of a compressive load, and this finding pointed out its good recoverability. These results suggested that the mechanical properties of Col-PEG/SF DN hydrogels were much better than those of the individual component hydrogel, and the $\mathrm{Col}_{5}-\mathrm{PEG}_{5} / \mathrm{SF}_{5}$ hydrogel showed greatest anti-compressive ability among these hydrogels.

In addition, the pore sizes, porosities and hydrophilicity of hydrogel scaffolds have major impacts on the survival, proliferation and differentiation of BMSCs. ${ }^{41}$ Therefore, the lyophilized hydrogels were examined under a SEM. As shown in Fig. 3, the $\mathrm{Col}_{5}-\mathrm{PEG}_{5} / \mathrm{SF}_{5}$ hydrogel showed high porosity and water absorption, and the pores sizes were 100-200 $\mu \mathrm{m}$, which are beneficial for the transportation of nutrients and metabolites. By contrast, the $\mathrm{Col}_{5}-\mathrm{PEG}_{5} / \mathrm{SF}_{10}$ hydrogel showed much lower porosity and water absorption, and the pores sizes were less than $100 \mu \mathrm{m}$, which could not provide a good microenvironment for BMSCs' survival and proliferation. Taking all conditions into consideration, it can be inferred that $\mathrm{Col}_{5}-\mathrm{PEG}_{5} / \mathrm{SF}_{5}$ possess excellent properties among the above-mentioned hydrogels, and it also exhibits an appropriate gelation rate, excellent biocompatibility and good mechanical properties. Furthermore, the biodegradation ability of the scaffold is another important factor for scaffolds in CTE. Therefore, the mass loss of the hydrogel was monitored for 4 weeks under different conditions. As shown in Fig. 3D, the mass loss of the hydrogel was the fastest when it was incubated in the solution with the highest collagenase concentration, and the mass loss was the lowest in PBS. After 4 weeks, the hydrogel lost nearly $65 \%$ weight in the collagenase solution of $200 \mathrm{U} \mathrm{mL}^{-1}$, while the hydrogel lost only $10 \%$ weight in PBS.

\subsection{Biocompatibility of the $\mathrm{Col}_{5}-\mathrm{PEG}_{5} / \mathrm{SF}_{5}$ hydrogel}

To evaluate the survival status of BMSCs within the $\mathrm{Col}_{5}-\mathrm{PEG}_{5} /$ $\mathrm{SF}_{5}$ hydrogel, WST assay and Live/Dead staining assay were performed at certain times. As shown in Fig. 4A, the viable BMSC density increased from the 1st day to the 7th day, and few dead BMSCs were displayed during this period, which illustrated that BMSCs were significantly proliferated within the $\mathrm{Col}_{5}-\mathrm{PEG}_{5} / \mathrm{SF}_{5}$ hydrogel. Furthermore, the 3D Live/Dead BMSC staining (Fig. 4B) further reflected that lots of BMSCs with good viability were well-distributed while few dead BMSCs were observed in the whole $\mathrm{Col}_{5}-\mathrm{PEG}_{5} / \mathrm{SF}_{5}$ hydrogel. In agreement with Live/Dead staining assay, the WST assay (Fig. 4C) result showed that the number of live BMSCs is notably increased from

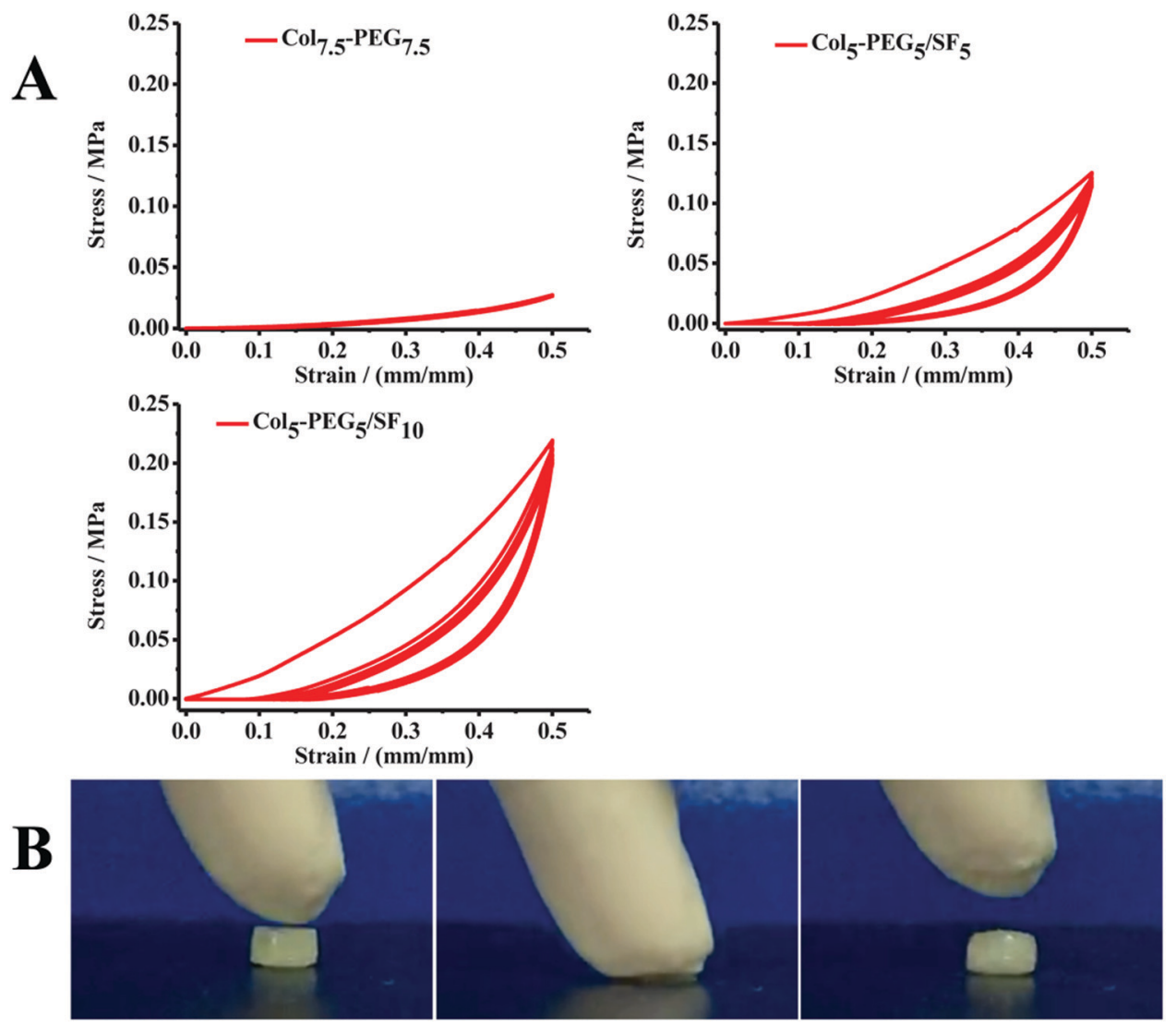

Fig. 2 Cyclic compression stress-strain curves of hydrogels (A); compression-recovery of the $\mathrm{Col}_{5}-\mathrm{PEG}_{5} / \mathrm{SF}_{5}$ hydrogel (B). 
A

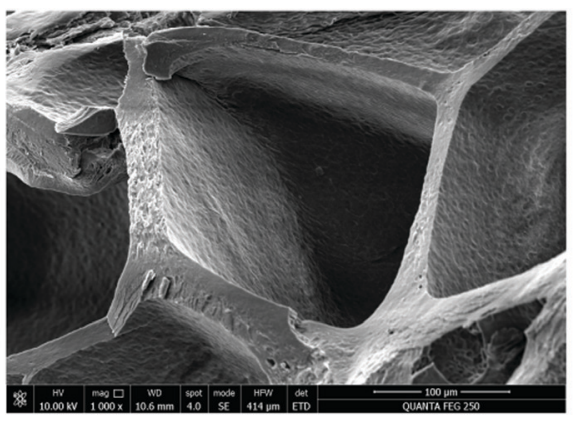

C

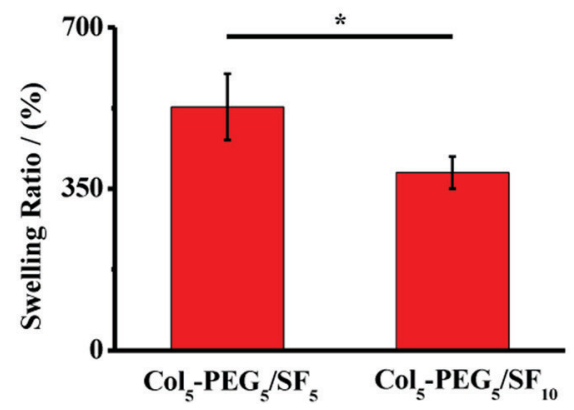

B

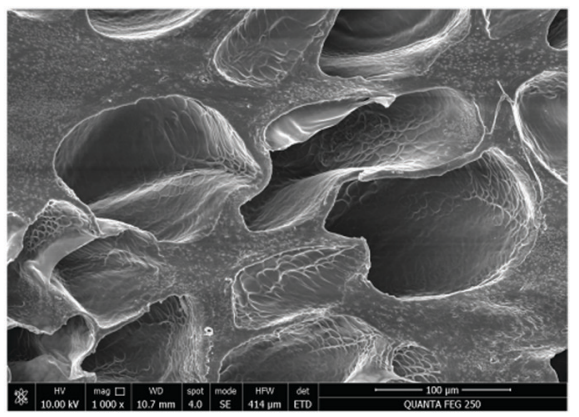

D

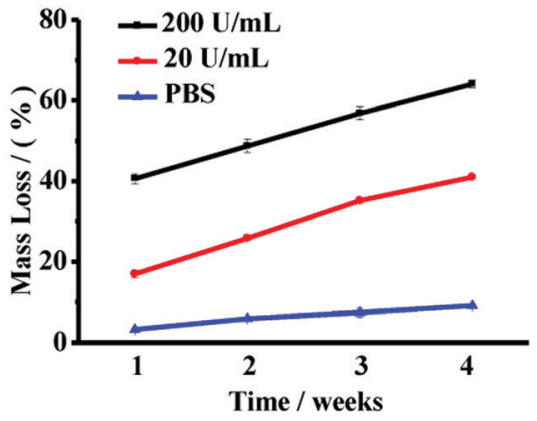

Fig. 3 Morphology of $\mathrm{Col}_{5}-\mathrm{PEG}_{5} / \mathrm{SF}_{5}(\mathrm{~A})$ and $\mathrm{Col}_{5}-\mathrm{PEG}_{5} / \mathrm{SF}_{10}(\mathrm{~B})$; swelling ratios of hydrogels $(\mathrm{C}, n=5)$; the degradation rate of $\mathrm{Col}_{5}-\mathrm{PEG}_{5} / \mathrm{SF}_{5}$ in the solution with 0 (PBS), 20 and $200 \mathrm{U} \mathrm{mL}^{-1}$ collagenase concentration (D, $\left.n=5\right)$.

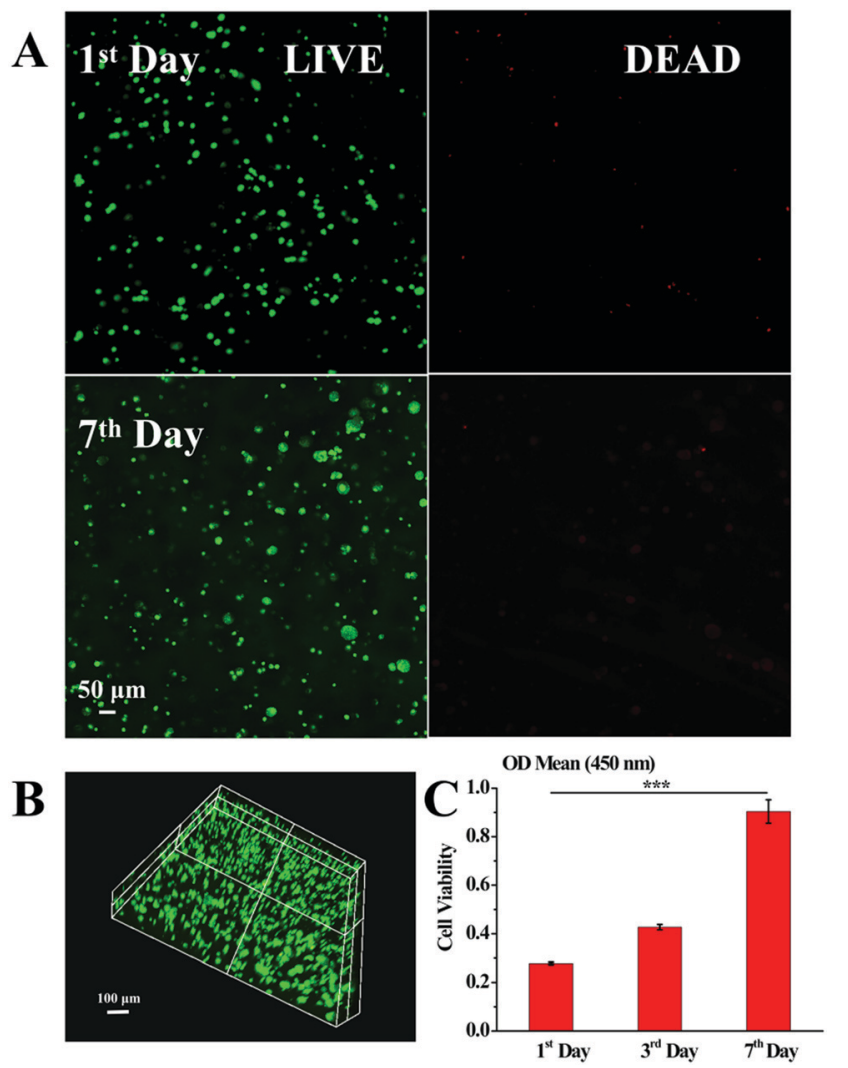

Fig. 4 The Live/Dead staining (A) and the 3D image of Live/Dead staining (B) of $\mathrm{BMSCs}$ within the $\mathrm{Col}_{5}-\mathrm{PEG}_{5} / \mathrm{SF}_{5}$ hydrogel; the proliferation of $\mathrm{BMSCs}$ within the $\mathrm{Col}_{5}-\mathrm{PEG}_{5} / \mathrm{SF}_{5}$ hydrogel at certain time point (C, $n=5)$. about 0.3 at the 1 st day to around 0.79 at the 7 th day. The above results consistently demonstrated that the $\mathrm{Col}_{5}-\mathrm{PEG}_{5} / \mathrm{SF}_{5}$ hydrogel supported the survival and proliferation of BMSCs.

3.3 In vitro evaluation of cartilage regeneration ability of the $\mathrm{Col}_{5}-\mathrm{PEG}_{5} / \mathrm{SF}_{5}$ hydrogel

To evaluate the chondrogenic differentiation of BMSCs within the $\mathrm{Col}_{5}-\mathrm{PEG}_{5} / \mathrm{SF}_{5}$ hydrogels, chondrogenic gene expression and chondrogenic matrix deposition were then analysed. As shown in Fig. 5, expression of chondrogenic genes, including Col II, AGG and Sox 9, was significantly higher at the 14th day than that at 0 day. Furthermore, at 28th day, the expression of gene was further regulated, which demonstrated that this hydrogel provided a good 3D microenvironment to improve the chondrogenic differentiation of BMSCs.

Furthermore, the chondrogenic differentiation ability of BMSCs within the $\mathrm{Col}_{5}-\mathrm{PEG}_{5} / \mathrm{SF}_{5}$ hydrogel was analysed by immunohistochemical analysis. Col II and AGG are cartilagespecific ECMs, and immunostaining of Col II and AGG is a commonly used method to assess the chondrogenesis or the quality of the regenerated cartilage. Fig. 6 shows that strong staining for Col II and AGG was detected in the hydrogel containing many chondrocyte-like cells after chondrogenic induction for 28 days, which indicated that this hydrogel supported cell proliferation and chondrogenesis.

\subsection{In vivo evaluation of cartilage regeneration ability of the $\mathrm{Col}_{5}-\mathrm{PEG}_{5} / \mathrm{SF}_{5}$ hydrogel}

To further evaluate the therapeutic efficacy of $\mathrm{Col}_{5}-\mathrm{PEG}_{5} / \mathrm{SF}_{5}$ hydrogels, samples of rabbit articular cartilage were harvested for 8 weeks and photographed. Fig. 7A shows the results at 8 weeks after surgery, and the defects were still visible to the 

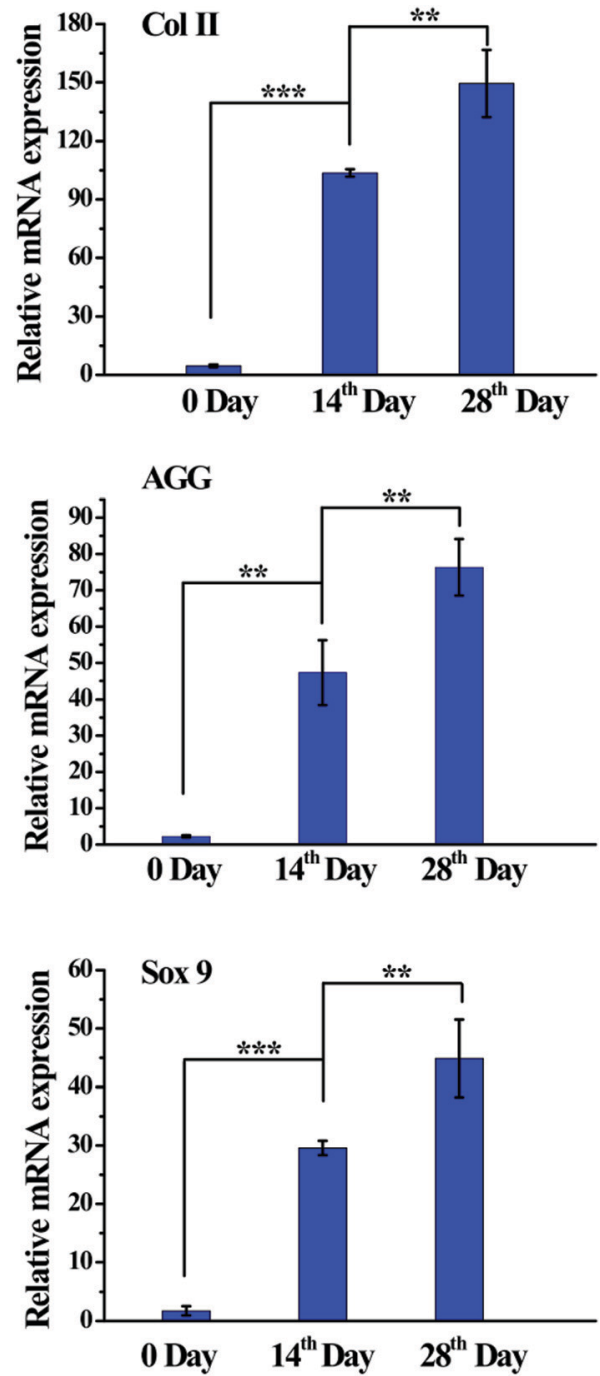

Fig. 5 Expression of the cartilage-specific genes Col II, AGG and Sox 9 within the BMSC-laden $\mathrm{Col}_{5}-\mathrm{PEG}_{5} / \mathrm{SF}_{5}$ hydrogel after culturing in chondrogenic differentiation medium for 0,14 and 28 days. The relative mRNA expression of undifferentiated BMSCs was set as $1(n=5)$.

naked eye for the control groups while the defects were mostly repaired in the hydrogel and $\mathrm{B}+\mathrm{T}$ hydrogel groups. Importantly, the regenerated cartilage integrated well with the adjacent host cartilage without boundaries in the $\mathrm{B}+\mathrm{T}$ hydrogel group; however, the boundary between the regenerated cartilage and surrounding normal ones was obviously observed in the hydrogel group. In addition, HE staining (Fig. 7B) further confirmed that the control group had the worst cartilage regeneration, which contained obvious defects with almost no new tissues. Oppositely, the $\mathrm{B}+\mathrm{T}$ hydrogel group had the best cartilage regeneration, in which the structural properties of generated cartilage were more similar to those of native cartilage. In the hydrogel group, the defect section was partially filled with thin fibrocartilage. The cartilage-specific ECM was investigated using Safranin-O/Fast Green (Fig. 7C) and toluidine blue staining (Fig. 7D). The results showed that more cartilage matrix had secreted in the $\mathrm{B}+\mathrm{T}$ hydrogel group compared with control and
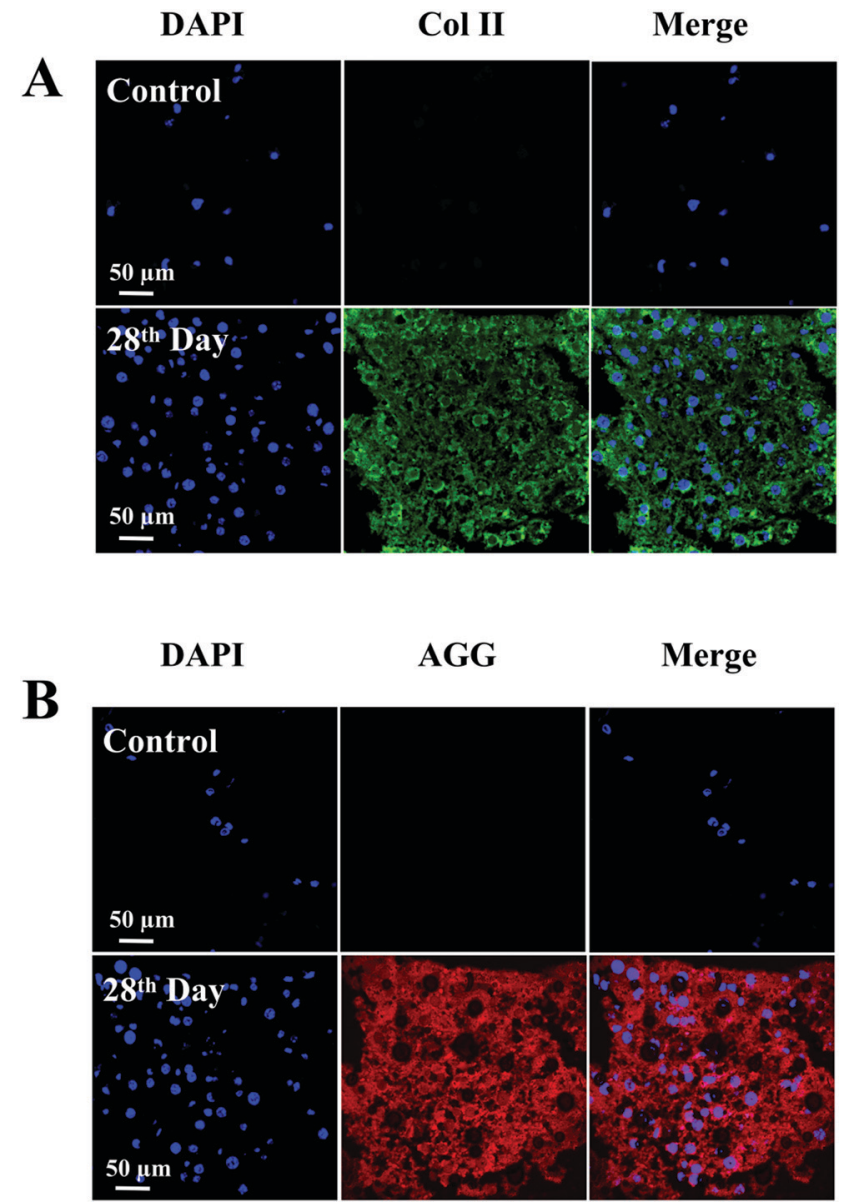

Fig. 6 Immunofluorescence staining of Col II (A) and AGG (B) of the $\mathrm{BMSC}$-laden $\mathrm{Col}_{5}-\mathrm{PEG}_{5} / \mathrm{SF}_{5}$ hydrogel after culturing in chondrogenic differentiation medium for 0 (control) and 28 days.

hydrogel groups. According to the modified Wakitani's histological scores (Fig. 8), the average scores for the $\mathrm{B}+\mathrm{T}$ hydrogel group were significantly higher than those for the hydrogel group and the control group. Taken together, these results revealed that a more hyaline-like cartilage was regenerated in the $\mathrm{B}+\mathrm{T}$ hydrogel group. In this research, an injectable $\mathrm{Col}_{5}-\mathrm{PEG}_{5} / \mathrm{SF}_{5}$ hydrogel was built for cartilage restoration. On the one hand, the $\mathrm{Col}_{5}-\mathrm{PEG}_{5} / \mathrm{SF}_{5}$ hydrogel had stronger mechanical properties to provide a better physical support for cartilage regeneration. On the other hand, because of the in situ fast-forming properties, the $\mathrm{Col}_{5}-\mathrm{PEG}_{5} / \mathrm{SF}_{5}$ hydrogel conveniently encapsulated with cells and growth factors and quickly formed in cartilage defects by a mini-invasive way, and the cells and growth factors could effectively promote the cartilage repair. In short, owing to the above advantages, the $\mathrm{B}+\mathrm{T}$ hydrogel group showed the best cartilage defect repair outcomes among these groups.

\section{Conclusion}

In this study, a Col/SF-based injectable DN hydrogel was fabricated through bioorthogonal reactions and ultrasonication 


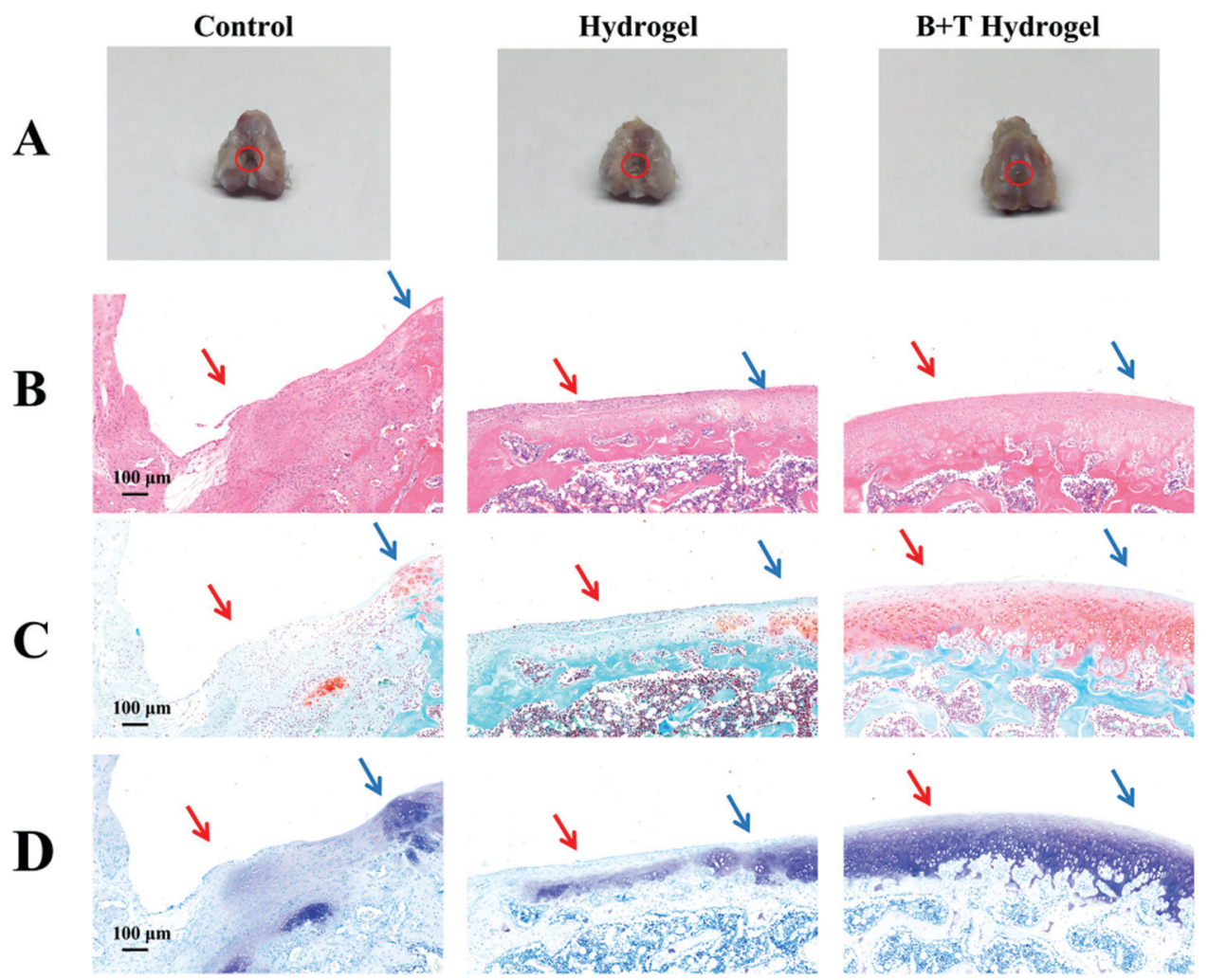

Fig. 7 Macroscopic images (A), HE (B), Safranin-O/Fast Green (C) and Toluidine blue (D) staining of the cartilage defects after 8 weeks' repair $(n=18$ ). The blue arrows indicate the original cartilage area, and the red arrows indicate the regenerative cartilage area.

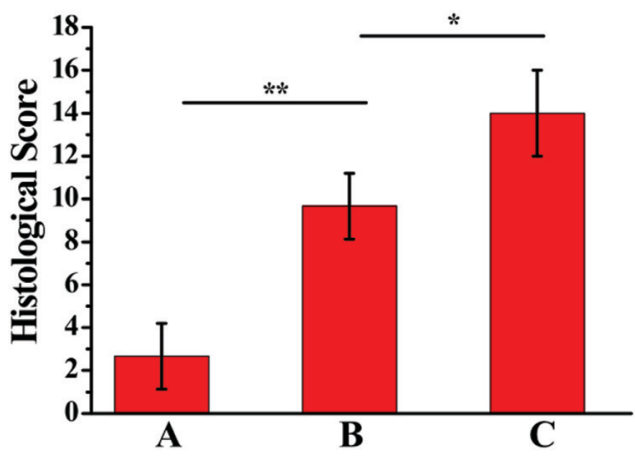

Fig. 8 Histological scores of control (A), hydrogel (B) and B + Thydrogel (C) groups for repaired cartilages at 8 weeks after surgery $(n=18)$.

for CTE. After optimizing the ratio of $\mathrm{Col}$ and $\mathrm{SF}$, the $\mathrm{Col}_{5}-\mathrm{PEG}_{5} /$ $\mathrm{SF}_{5}$ hydrogel with a high gelation rate, great biocompatibility and good mechanical properties was finally chosen for further experiments. The experimental results illustrated that the mechanical properties obviously improved and the $\mathrm{Col}_{5}-\mathrm{PEG}_{5} / \mathrm{SF}_{5}$ hydrogel could be used for cartilage defect regeneration. In vivo experiments further indicated that the $\mathrm{Col}_{5}-\mathrm{PEG}_{5} / \mathrm{SF}_{5}$ hydrogel encapsulated with BMSCs and TGF- $\beta 1$ achieved integrative hyaline cartilage regeneration. Overall, the $\mathrm{Col}_{5}-\mathrm{PEG}_{5} / \mathrm{SF}_{5}$ hydrogel expanded the applications of $\mathrm{Col}$ and is a potential candidate for cartilage tissue engineering.

\section{Conflicts of interest}

There are no conflicts to declare.

\section{Acknowledgements}

This work was supported by the Strategic Priority Research Program of Chinese Academy of Science (XDA16040700), the National Natural Science Foundation of China (No. 31971326), the Science Foundation of Jiangsu Province (No. BE2018665), the China Postdoctoral Science Foundation (2020M681731) and the Science and Technology Foundation of Wuhan (2020020601012326).

\section{Notes and references}

1 G. M. Williams, S. M. Klisch and R. L. Sah, Pediatr. Res., 2008, 63, 527-534.

2 S. Camarero-Espinosa, B. Rothen-Rutishauser, E. J. Foster and C. Weder, Biomater. Sci., 2016, 4, 734-767.

3 A. J. Sophia Fox, A. Bedi and S. A. Rodeo, Sports Health, 2009, 1, 461-468.

4 D. J. Huey, J. C. Hu and K. A. Athanasiou, Science, 2012, 338, 917-921.

5 T. A. Ahmed and M. T. Hincke, Tissue Eng., Part B, 2010, 16, 305-329.

6 D. A. Grande, A. S. Breitbart, J. Mason, C. Paulino, J. Laser and R. E. Schwartz, Clin. Orthop. Relat. Res., 1999, S176-S185. 
7 B. J. Huang, J. C. Hu and K. A. Athanasiou, Biomaterials, 2016, 98, 1-22.

8 R. Langer and J. P. Vacanti, Science, 1993, 260, 920-926.

9 E. A. Makris, A. H. Gomoll, K. N. Malizos, J. C. Hu and K. A. Athanasiou, Nat. Rev. Rheumatol., 2015, 11, 21-34.

10 J. S. Temenoff and A. G. Mikos, Biomaterials, 2000, 21, 431-440.

11 M. Krampera, G. Pizzolo, G. Aprili and M. Franchini, Bone, 2006, 39, 678-683.

12 P. K. Gupta, A. K. Das, A. Chullikana and A. S. Majumdar, Stem Cell Res. Ther., 2012, 3, 25.

13 A. Inui, T. Iwakura and A. H. Reddi, Cells, 2012, 1, 994-1009.

14 M. J. Chen, J. P. Whiteley, C. P. Please, F. Ehlicke, S. L. Waters and H. M. Byrne, J. Tissue Eng., 2019, 10, 2041731419842431.

15 B. J. Huang, J. C. Hu and K. A. Athanasiou, Biomaterials, 2016, 98, 1-22.

16 H. X. Le, W. G. Xu, X. L. Zhuang, F. Chang, Y. N. Wang and J. X. Ding, J. Tissue Eng., 2019, 11, 2041731420943839.

17 M. H. Zheng, C. Willers, L. Kirilak, P. Yates, J. Xu, D. Wood and A. Shimmin, Tissue Eng., 2007, 13, 737-746.

18 D. C. Crawford, C. M. Heveran, W. D. Cannon, L. F. Foo and H. G. Potter, Am. J. Sports Med., 2009, 37, 1334-1343.

19 T. R. Niethammer, M. F. Pietschmann, A. Horng, B. P. Rossbach, A. Ficklscherer, V. Jansson and P. E. Müller, Knee Surg. Sports Traumatol. Arthrosc. Off. J. ESSKA, 2014, 22, 1329-1336.

20 U. Schneider, L. Rackwitz, S. Andereya, F. Fensky, J. Reichert, I. Löer, T. Barthel, M. Rudert and U. Nöth, Am. J. Sports Med., 2011, 39, 2558-2565.

21 X. Zhang, W. Zhang and M. Yang, Curr. Stem Cell Res. Ther., 2018, 13, 497-516.

22 O. Jeznach, D. Kolbuk and P. Sajkiewicz, J. Biomed. Mater. Res., Part A, 2018, 106, 2762-2776.

23 C. M. Madl and S. C. Heilshorn, Adv. Funct. Mater., 2018, 28, 1706046.
24 Y. J. Zhang, H. Chen, T. T. Zhang, Y. Zan, T. Y. Ni, M. Liu and R. J. Pei, Biomater. Sci., 2018, 6, 2578-2581.

25 K. Lang and J. W. Chin, ACS Chem. Biol., 2014, 9, 16-20.

26 C. M. Madl and S. C. Heilshorn, Adv. Funct. Mater., 2018, 28, 1706046.

27 D. L. Alge, M. A. Azagarsamy and D. F. Donohue, Biomacromolecules, 2013, 14, 949-953.

28 R. M. Desai, S. T. Koshy, S. A. Hilderbrand, D. J. Mooney and N. S. Joshi, Biomaterials, 2015, 50, 30-37.

29 D. Eyrich, F. Brandl, B. Appel, H. Wiese, G. Maier, M. Wenzel, R. Staudenmaier, A. Goepferich and T. Blunk, Biomaterials, 2007, 28, 55-65.

30 J. P. Gong, Y. Katsuyama, T. Kurokawa and Y. Osada, Adv. Mater., 2003, 15, 1155-1158.

31 Z. J. Wang, C. P. Xiang, X. Yao, P. L. Floch, J. Mendez and Z. G. Suo, Proc. Natl. Acad. Sci. U. S. A., 2019, 116, 5967-5972.

32 M. Voga, N. Drnovsek, S. Novak and G. Majdic, J. Tissue Eng., 2019, 10, 2041731419835056.

33 A. T. Nguyen, Q. L. Huang, Z. Yang, N. Lin, G. Xu and X. Y. Liu, Small, 2015, 11, 1039-1054.

34 B. B. Mandal, S. H. Park, E. S. Gil and D. L. Kaplan, Biomaterials, 2011, 32, 639-651.

35 Y. J. Zhang, Y. Cao, L. W. Zhang, H. B. Zhao, T. Y. Ni, Y. S. Liu, Z. An, M. Liu and R. J. Pei, J. Mater. Chem. B, 2020, 8, 5845-5848.

36 Y. J. Zhang, Y. Cao, H. B. Zhao, L. W. Zhang, T. Y. Ni, Y. S. Liu, Z. An, M. Liu and R. J. Pei, J. Mater. Chem. B, 2020, 8, 4237-4244.

37 X. Hu, D. Kaplan and P. Cebe, Macromolecules, 2006, 39, 6161-6170.

38 Q. Lu, B. Zhang, M. Li, B. Zuo, D. L. Kaplan, Y. Huang and H. Zhu, Biomacromolecules, 2011, 12, 1080-1086.

39 B. D. Lawrence, F. Omenetto, K. Chui and D. L. Kaplan, J. Mater. Sci., 2008, 43, 6967-6985.

40 Y. Wang, U. J. Kim, D. J. Blasioli, H. J. Kim and D. L. Kaplan, Biomaterials, 2005, 26, 7082-7094.

41 D. Seliktar, Science, 2012, 336, 1124-1128. 